$07,12,13$

\title{
Сравнительный анализ деформационных полей в слоях метаморфных ступенчатых буферов различного дизайна
}

\author{
(c) А.Н. Алёшин ${ }^{1}$, А.С. Бугаев ${ }^{1}$, О.А. Рубан ${ }^{1}$, Н.Ю. Табачкова ${ }^{2}$, И.В. Щетинин ${ }^{2}$ \\ ${ }^{1}$ Институт сверхвысокочастотной полупроводниковой электроники РАН, \\ Москва, Россия \\ ${ }^{2}$ Национальный исследовательский технологический университет „МИСиС“, \\ Москва, Россия \\ E-mail: a.n.aleshin@mail.ru
}

(Поступила в Редакцию 9 марта 2017 г.)

\begin{abstract}
Методом построения карт обратного пространства, полученных с помощью трехосевой рентгеновской дифрактометрии, и на основе линейной теории упругости получены пространственные распределения остаточных упругих деформаций в слоях двух метамофных ступенчатых буферов различного дизайна, выращенных методом молекулярно-лучевой эпитаксии на основе тройных растворов $\operatorname{In}_{x} \mathrm{Al}_{1-x}$ As на подложках (001) GaAs. Разница в дизайне буферов обеспечивала образование в каждой из гетероструктур бездислокационного слоя с различной толщиной, что явилось основным базисом данного исследования. Показано, что, несмотря на различный дизайн метаморфных ступенчатых буферов, характер деформационных полей в них один и тот же, а остаточные упругие деформации в финальных элементах обоих буферов с учетом поправки на эффект деформационного упрочнения подчиняются тому же феноменологическому закону, который описывает процесс структурной релаксации в однослойных гетероструктурах.
\end{abstract}

Работа выполнена при частичной финансовой поддержке РФФИ (гранты № 16-07-00187 А и 16-29-03033 офи_м).

DOI: 10.21883/FTT.2017.10.44965.068

\section{1. Введение}

Одной из актуальных проблем современного материаловедения является изучение процесса структурной релаксации в слоях метаморфного буфера (ММ-буфера), используемого в гетероструктурах, создаваемых, в частности, для приборов сверхвысокочастотной полупроводниковой электроники, например в транзисторах с высокой подвижностью электронов (high electron mobility transistors, HEMT). ММ-буфер является демпфирующим элементом конструкции гетероструктуры (например, гетероструктуры AllnAs/AlGaAs). Необходимость его присутствия обусловлена тем, что параметр решетки активных слоев транзистора (квантовой ямы) превышает параметр подложки GaAs, на которой обычно выращивается гетероструктура. ММ-буфер представляет собой переходную область с пространственным изменением периода решетки, что в случае гетероструктур AlInAs/AlGaAs достигается путем изменения содержания In. Дизайн ММ-буфера может быть различным, например ступенчатым [1-3] или линейным [2,3]. При выращивании ММ-буфера одновременно с ростом эпитаксиальных слоев происходит их структурная релаксация, которая сопровождается генерацией дислокаций несоответствия и распространением прорастающих дислокаций (представляющих собой дислокационные петли, замкнутые на дислокации несоответствия [4]) в верхние слои гетероструктуры вплоть до активных слоев НЕМТ. В технологии создания гетероструктур наибольшее распространение по- лучили многослойные ступенчатые ММ-буферы ввиду относительной простоты их изготовления. Часто в ступенчатом ММ-буфере в качестве дополнительных элементов конструкции присутствуют инверсная ступень или залечивающий слой [5]. Наличие ММ-буфера предохраняет распространение прорастающих дислокаций в активные слои прибора. Эта цель достигается за счет образования в верхней части ММ-буфера слоя, свободного от прорастающих дислокаций, который в свою очередь служит платформой для создания инверсной ступени или залечивающего слоя. Теоретическое обоснование возможности образования бездислокационного слоя в системе переменного состава (т.е. с изменяющимся периодом решетки) было дано в [6]. Данная теоретическая модель основана на стремлении системы к равновесию, которое наиболее полно достигается при образовании в верхней части буфера бездислокационного слоя, что обеспечивает значительный энергетический выигрыш $[3,6]$. Образование бездислокационного слоя является одной из стадий процесса структурной релаксации, происходящей при выращивании ММ-буфера, а уровень остаточных упругих деформаций в этом слое представляет собой важнейшую характеристику всей конструкции гетероструктуры. Выявление основных закономерностей, лежащих в основе формирования бездислокационного слоя ММ-буфера, - актуальная задача, решение которой важно для разработки физических основ технологии создания гетероструктур. 
Наиболее подробно процесс структурной релаксации был изучен для однослойных гетероструктур состава $\mathrm{In}_{x} \mathrm{Ga}_{1-x} \mathrm{As}$, выращенных на подложках GaAs с ориентацией $(001)[7,8]$. Было установлено, что в однослойных гетероструктурах структурная релаксация характеризуется тремя стадиями: медленной, быстрой и финальной стадией насыщения. Медленная стадия характеризуется проникновением решеточных дислокаций из подложки в эпитаксиальный слой и их искривлением, что приводит к образованию на межфазной границе дислокаций несоответствия. Быстрая стадия - стадия мультипликации дислокаций несоответствия и скольжения их дислокационных петель; она протекает при толщине эпитаксиального слоя порядка $100-1000 \mathrm{~nm}$. Третья стадия заключается в торможении процесса мультипликации дислокаций несоответствия и скольжения дислокационных петель, что обусловлено эффектом деформационного упрочнения. Для быстрой стадии структурной релаксации был получен ряд численных соотношений, устанавливающих связь между величиной остаточных упругих деформаций и толщиной эпитаксиального слоя. Так, Данстэн с сотрудниками в работах $[9,10]$ установил, что между величиной остаточной упругой деформации и толщиной гетероэпитаксиального слоя имеется обратно пропорциональная зависимость. Эта зависимость была объяснена особенностями поведения дислокаций несоответствия в приграничном (в области подложки) слое на основе геометрического подхода [11], базирующегося на существовании предельной плотности дислокаций несоответствия, превышение которой приводит к пластической деформации. Авторами [12] по результатам собственных исследований, а также на основе экспериментальных данных, приведенных в [13-15], было установлено, что связь между остаточными упругими деформациями и толщинами слоев в однослойных гетероструктурах подчиняется обратной квадратно-корневой зависимости, что свидетельствует о существовании некоторого энергетического предела, выше которого дальнейшее упругое сжатие решетки эпитаксиального слоя невозможно. Для обоих подходов были найдены значения феноменологических постоянных, позволяющих численно выразить остаточную упругую деформацию в зависимости от толщины эпитаксиального слоя.

Цель настоящей работы состоит в выявлении возможности применения феноменологического подхода для описания процесса структурной релаксации в многослойных тонкопленочных системах, в частности в слоях ступенчатого ММ-буфера, и нахождении численных критериев, характеризующих образование бездислокационного слоя. Решение этой задачи, по нашему мнению, возможно путем сравнительного анализа деформационных полей в гетероструктурах, в которых используются ступенчатые ММ-буферы различного дизайна. В данной работе разница в дизайне двух ММ-буферов заключалась в разной толщине ступеней, а также в различной толщине бездислокационного слоя. Ступени каждого из ММ-буферов представляли собой эпитак- сиальные слои состава $\operatorname{In}_{x} \mathrm{Al}_{1-x}$ As. В работе использованы методы построения карт обратного пространства и просвечивающая электронная микроскопия (ПЭМ). Далее с целью унификации обозначений для гетероструктур с ММ-буфером будем использовать аббревиатуру MHEMT (metamorphic HEMT). Одну из изученных гетероструктур с ММ-буфером, в котором в качестве финального элемента конструкции использовался залечивающий слой, будем обозначать как MHEMT 1, a другую гетероструктуру, у которой в ММ-буфере имелась инверсная ступень, - как MHЕМТ 2.

\section{2. Эксперимент}

Две MHЕМТ, каждая из которых имела ступенчатый ММ-буфер, были выращены методом молекулярно-лучевой эпитаксии (МЛЭ) на установке RIBER 32 MBE. Oбе MHEMT состояли из активных слоев InAlAs/InGaAs/ InAlAs и шести ступенчатых ММ-буферов с близким мольным содержанием индия $X_{\mathrm{In}}$ в активных слоях, равным 0.39 (МНЕМТ 1) и 0.394 (МНЕМТ 2). МЛЭ была выполнена при постоянной температуре подложки. В случае МНЕМТ 1 использовалась стандартная полуизолирующая подложка (001) GaAs, а гетероструктура MHEMT 2 была выращена на вицинальной поверхности подложки GaAs с отклонением от плоскости (001), равным $2^{\circ}$. Изменение мольной доли In при переходе от одной ступени ММ-буфера к другой для первых пяти ступеней обеих МНЕМТ было выполнено без прерывания роста при постоянной температуре источника алюминия. На этой стадии эпитаксиального роста толщина каждой из ступеней ММ-буфера в случае MHEMT 1 равнялась $0.1 \mu \mathrm{m}$, а в случае МНЕМТ $2-0.2 \mu \mathrm{m}$. Температура подложки во время роста для МНЕМТ 1 составляла $380^{\circ} \mathrm{C}$, а для МНЕМТ $2-400^{\circ} \mathrm{C}$. Барьерные слои обеих МНЕМТ были выращены при более высокой температуре подложки: при 480 и $500^{\circ} \mathrm{C}$ соответственно. Скорость роста эпитаксиальных слоев равнялась $0.5 \mu \mathrm{m} \cdot \mathrm{h}^{-1}$. При выращивании слоя 6 (залечивающего слоя) в МНЕМТ 1 перед началом его роста по технологическим причинам процесс роста был остановлен на $2 \mathrm{~min}$. Процесс роста прерывался также на $5 \mathrm{~min}$ в обеих МНЕМТ при переходе к более высокой температуре роста нижних барьерных слоев. Состав элементов конструкции гетероструктур контролировался температурой молекулярных источников In, Al, As, $\mathrm{Ga}$ на основе калибровки температурной зависимости молекулярных потоков. Общей чертой конструкции обеих МНЕМТ было равенство толщин залечивающего слоя (MHEMT 1) и инверсной ступени (MHEMT 2), а также равенство толщин нижних барьерных слоев. Технологические детали эпитаксиального роста, а также основные характеристики конструкционных элементов в обеих МНЕМТ приведены в табл. 1.

Рентгеновская съемка была выполнена в трехосевой геометрии с использованием прецизионного дифракто- 
Таблица 1. Основные ростовые характеристики исследованных гетероструктур

\begin{tabular}{|c|c|c|c|c|c|}
\hline Гетероструктура & $\begin{array}{l}\text { Номер } \\
\text { слоя }\end{array}$ & $X_{\mathrm{In}}$ & $\begin{array}{l}\text { Толщина } \\
\text { слоя, } \mu \mathrm{m}\end{array}$ & Назначение слоя & $\begin{array}{c}\text { Температура } \\
\text { подложки, }{ }^{\circ} \mathrm{C}\end{array}$ \\
\hline MHEMT 1 & $\begin{array}{l}1 \\
2 \\
3 \\
4 \\
5 \\
6 \\
7\end{array}$ & $\begin{array}{l}0.15 \\
0.23 \\
0.29 \\
0.35 \\
0.39 \\
0.39 \\
0.39\end{array}$ & $\begin{array}{l}0.1 \\
0.1 \\
0.1 \\
0.1 \\
0.1 \\
0.2 \\
0.2\end{array}$ & $\begin{array}{l}\text { Первая ступень ММ-буфера } \\
\text { Вторая ступень ММ-буфера } \\
\text { Третья ступень ММ-буфера } \\
\text { Четвертая ступень ММ-буфера } \\
\text { Пятая ступень ММ-буфера } \\
\text { Залечивающий слой } \\
\text { Барьерный слой }\end{array}$ & $\begin{array}{l}380 \\
380 \\
380 \\
380 \\
380 \\
380 \\
480\end{array}$ \\
\hline MHEMT 2 & $\begin{array}{l}1 \\
2 \\
3 \\
4 \\
5 \\
6 \\
7\end{array}$ & $\begin{array}{l}0.10 \\
0.20 \\
0.30 \\
0.39 \\
0.48 \\
0.394 \\
0.394\end{array}$ & $\begin{array}{l}0.2 \\
0.2 \\
0.2 \\
0.2 \\
0.2 \\
0.2 \\
0.2\end{array}$ & $\begin{array}{l}\text { Первая ступень ММ-буфера } \\
\text { Вторая ступень ММ-буфера } \\
\text { Третья ступень ММ-буфера } \\
\text { Четвертая ступень ММ-буфера } \\
\text { Пятая ступень ММ-буфера } \\
\text { Инверсная ступень } \\
\text { Барьерный слой }\end{array}$ & $\begin{array}{l}400 \\
400 \\
400 \\
400 \\
400 \\
400 \\
500\end{array}$ \\
\hline
\end{tabular}

метра SmartLab 9kW в пошаговом режиме в излучении $\mathrm{Cu} K_{\alpha_{1}}$. В качестве кристалла-анализатора был использован монокристалл $\mathrm{Ge}$ с ориентацией (002). Съемка велась в двух отражениях 004 и 224 (в положении скользящего отражения) в режиме так называемого $\omega-2 \theta$-сканирования, которое заключается в съемке образца по методу Брэгга-Брентано при различных исходных угловых отклонениях подложки от угла Брэгга, что достигается за счет поворота образца на фиксированный угол $\Delta \omega$ относительно одной из кристаллографических осей типа $\langle 110\rangle$. Во время съемки проводилась регистрация дифрагированного рентгеновского излучения для выбранного отражения путем сканирования вдоль вектора рассеяния $H_{h k l}: H_{001}$ (симметричная съемка) и $H_{224}$ (асимметричная съемка). При симметричной съемке (отражение 004) начальное положение образца соответствовало положению подложки под углом Брэгга $(\Delta \omega=0)$. При асимметричной съемке (отражение 224) варьировались значения $h k l$, что позволило разложить вектор $H_{224}$ по направлениям обратного пространства [001] и [110]. Более подробно режим $\omega-2 \theta$-сканирования описан в $[16,17]$. Образец для рентгеновской съемки имел размер $1.5 \times 1.5 \mathrm{~cm}$.

\section{3. Экспериментальные результаты}

3.1. Характеристика микроструктуры MHЕМТ 1 и 2. Исследование микроструктуры МНЕМТ было выполнено с помощью электронного микроскопа Jeol JEM 2010 при ускоряющем напряжении $200 \mathrm{kV}$. Результаты структурной аттестации (светлопольные поперечные электронно-микроскопические изображения MHEMT 1 и 2) показаны на рис. 1. Видно, что в обеих МНЕМТ в области финальных элементов конструкции прорастающие дислокации отсутствуют. В МНЕМТ 1 (рис. 1,a) бездислокационная область охватывает слои 5-7. Несмотря на прерывание роста

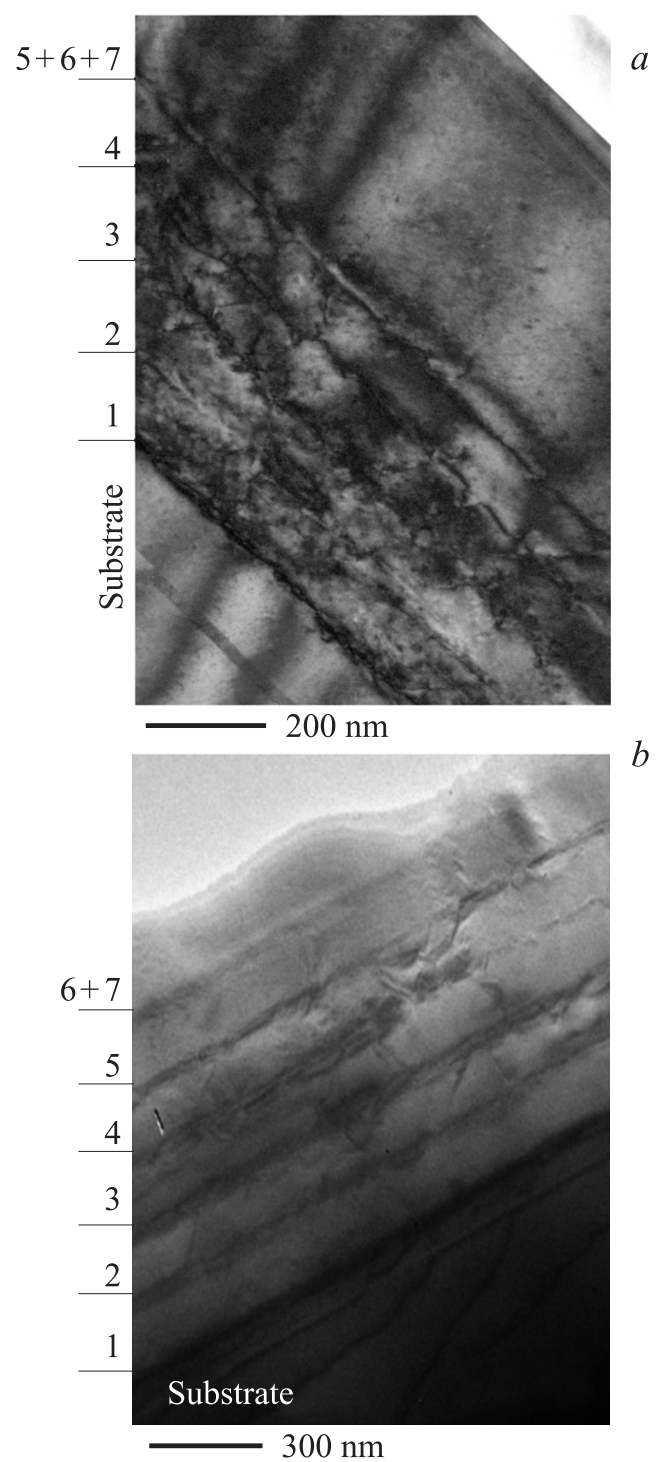

Рис. 1. Поперечные светлопольные электронно-микроскопические изображения изученных гетероструктур: MHEMT 1 (a) и MHEMT $2(b)$. Цифрами обозначены номера слоев. 
при выращивании верхних слоев МНЕМТ 1 (как указывалось выше, в случае МНЕМТ 1 при переходе к слоям 6 и 7 эпитаксиальный рост прерывался), в верхней бездислокационной области отсутствуют межфазные границы; следовательно, эту область, учитывая, что слои 5-7 имеют один и тот же состав, можно рассматривать как одну фазу. В МНЕМТ 2 бездислокационным слоем ММ-буфера является слой 5 с мольной долей In $X_{\text {In }}=0.48$. Отсутствие дислокаций в инверсной ступени (слой 6) и в нижнем барьерном слое (слой 7) - слоях, выращенных на бездислокационном слое, - достигается за счет правильного подбора содержания In в этих слоях ( $X_{\text {In }}$ в них составляет 0.394). Роль этих конструкционных элементов в формировании деформационного поля в МНЕМТ 2 обсуждается далее. Для последующего изложения важно отметить, что, согласно ПЭМ, инверсная ступень и нижний барьерный слой представляют собой единый структурный элемент.

3.2. Карты обратного пространства и их о бр аб о тка. Режим $\omega-2 \theta$-сканирования позволяет выявить неосновные рентгеновские максимумы для всех эпитаксиальных слоев ММ-буферов в обеих МНЕМТ и на этой основе построить так называемые карты обратного пространства, представляющие собой набор линий равной интенсивности дифрагированного рентгеновского излучения, включая области пучностей (рентгеновских максимумов), с указанием их расположения в обратном пространстве. Актуальными осями обратного пространства для эпитаксиальных слоев гетероструктуры, выращенной на подложке (001) GaAs, являются ось [110] (ось $Y$ ) и ось [001] (ось Z). За начало координат обратного пространства принимается точка, задаваемая вектором рассеяния от подложки - основного рентгеновского максимума. Координаты неосновных рентгеновских максимумов определяются векторами $q_{110}\left(q_{y}\right)$ и $q_{001}\left(q_{z}\right)$, которые представляют собой отклонения (вдоль соответствующих осей обратного пространства) от положения основного рентгеновского максимума $H_{h k l}^{0}$, возникающего от подложки. Величины $q_{001}$ и $q_{110}$ позволяют определить вертикальный и латеральный периоды решетки всех эпитаксиальных слоев, образующих гетероструктуру. Карты обратного пространства для изученных МНЕМТ для отражений 004 и 224 показаны на рис. 2 (для МНЕМТ 1) и рис. 3 (для МНЕМТ 2). Карты обратного пространства для MHEMT 1 имеют пять неосновных рентгеновских максимумов, которые в соответствии с ростом величины $\left|q_{z}\right|$ можно приписать слоям 1, 2, 3, 4 и 5(6,7). Наличие одного (пятого по счету) рефлекса свидетельствует, что слои 5-7 в МНЕМТ 1 являются в структурном отношении одинаковыми, это согласуется с данными электронно-микроскопических исследований. Карты обратного пространства для МНЕМТ 2 имеют более сложное строение благодаря частичному перекрытию рефлексов, а также отсутствию четко выраженного рефлекса для слоя 4. На основании значений вектора $\left|q_{z}\right|$ и исходя из результатов исследования микроструктуры этой
MHEMT (рис. $1, b$ ) можно сделать вывод, что слои 6 и 7 (инверсная ступень и нижний барьерный слой) характеризуются одним сильным рефлексом, который на картах обратного пространства находится между рефлексом, соответствующим слою 3 , и областью дифрагированного рентгеновского излучения, соответствующей слою 4. Близость концентрации In в слоях 4 и 6(7) затрудняет четкое выделение отдельного рентгеновского пика для слоя 4 на фоне сильного рентгеновского максимума, соответствующего слоям 6(7). С целью нахождения позиций всех рентгеновских максимумов на карте обратного пространства, построенной на основе отражения 004, мы использовали процедуру моделирования скана рентгеновского отраженного излучения (аналога кривой качания) набором гауссиан [18]. При этом мы считали, что слои 6 и 7 характеризуются одним гауссианом. Таким образом, количество гауссиан ограничивалось числом 6. Такой подход облегчает процедуру обработки карты обратного пространства для отражения 004 в случае MHEMT 2. Разложение сканов дифрагированного рентгеновского излучения на гауссианы вдоль оси обратного пространства $Z$ (в центральной части карты обратного пространства, т.е. при малых значениях вектора $q_{y}^{004}$ ) позволило определить величины $q_{z}^{004}$ для слоев 3,4 и $6(7)$. В качестве значений векторов $q_{z}^{004}$ для указанных слоев были приняты медианные значения соответствующих гауссиан. Аналогичный метод - представление отдельного рентгеновского максимума на картах обратного пространства для отражения 004 с помощью функции Гаусса - был использован нами для определения векторов $q_{y}^{004}$ (разложение вдоль оси $Y$ ). Координаты четко обозначенных рентгеновских максимумов определялись путем нахождения точки с максимальным значением интенсивности дифрагированного рентгеновского излучения. Для этой цели использовалась специальная опция компьютерной программы Origin 15. Значения векторов $q_{z}^{004}, q_{y}^{004}$ и $q_{y}^{224}$ и для всех слоев МНЕМТ 1 и 2 (за исключением значения вектора $q_{y}^{224}$ для слоя 4 в МНЕМТ 2) представлены в табл. 2.

Таблица 2. Векторы обратного пространства, полученные для отражений 004 и 224

\begin{tabular}{|c|c|c|c|c|}
\hline Гетероструктура & Слой & $\begin{array}{c}q_{z}^{004} \\
\mu \mathrm{m}^{-1}\end{array}$ & $\begin{array}{c}q_{y}^{004} \\
\mu \mathrm{m}^{-1}\end{array}$ & $\begin{array}{c}q_{y}^{224} \\
\mu \mathrm{m}^{-1}\end{array}$ \\
\hline MHEMT 1 & $\begin{array}{c}1 \\
2 \\
3 \\
4 \\
5(6,7)\end{array}$ & $\begin{array}{l}-75.36 \\
-111.45 \\
-139.32 \\
-165.35 \\
-204.70\end{array}$ & $\begin{array}{l}-4.90 \\
-5.00 \\
-5.03 \\
-5.114 \\
-5.00\end{array}$ & $\begin{array}{l}-44.93 \\
-67.39 \\
-86.96 \\
-110.07 \\
-111.71\end{array}$ \\
\hline MHEMT 2 & $\begin{array}{c}1 \\
2 \\
3 \\
4 \\
5 \\
6(7)\end{array}$ & $\begin{array}{l}-61.38 \\
-107.42 \\
-150.66 \\
-187.08 \\
-256.04 \\
-176.78\end{array}$ & $\begin{array}{r}2.53 \\
1.27 \\
0.51 \\
-10.75 \\
-12.67 \\
-12.67\end{array}$ & $\begin{array}{c}-26.84 \\
-53.73 \\
-87.33 \\
- \\
-140.30 \\
-140.30\end{array}$ \\
\hline
\end{tabular}



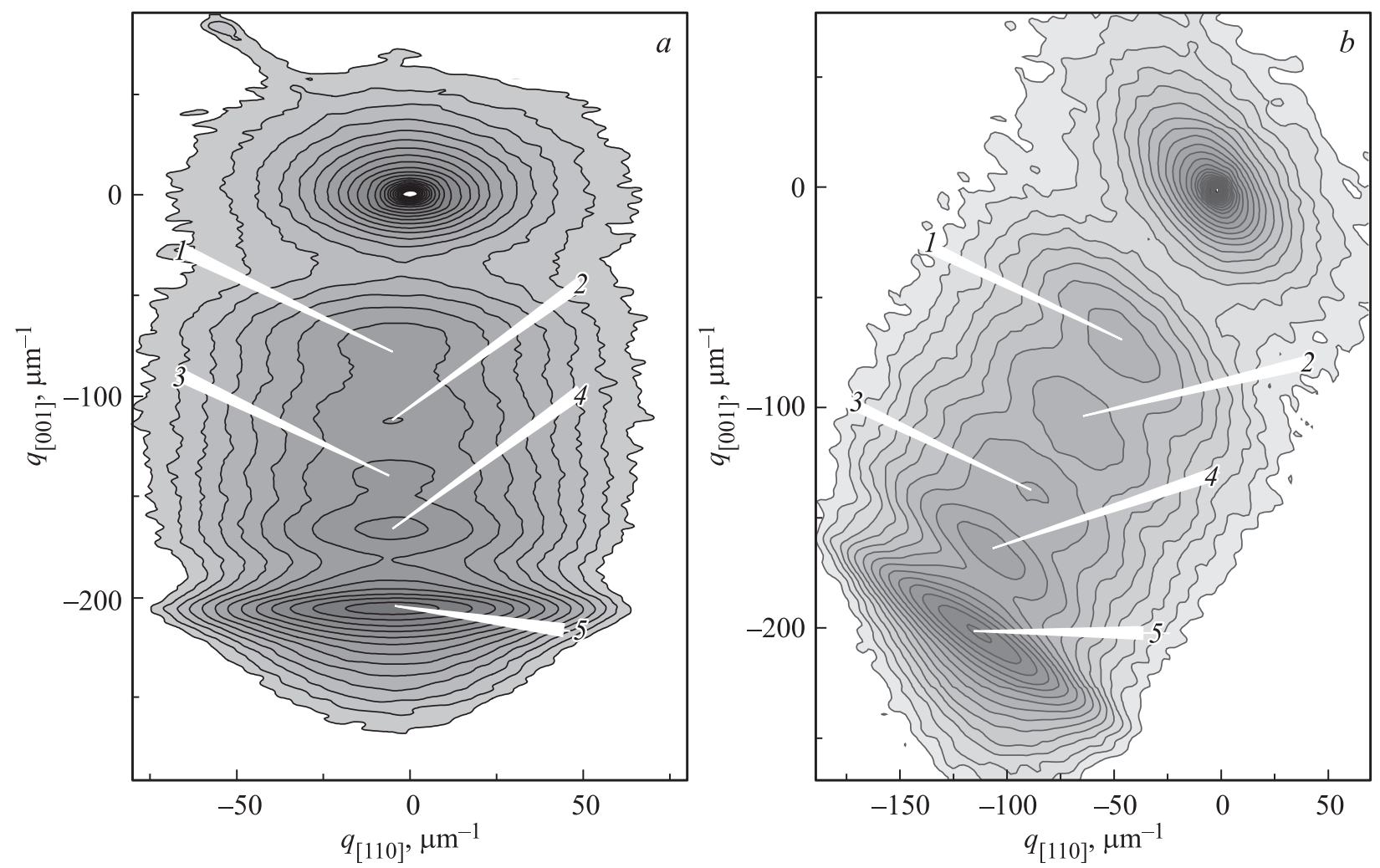

Рис. 2. Карты обратного пространства для МНЕМТ 1, полученные на основе отражений $004(a)$ и $224(b)$. Цифрами обозначены номера эпитаксиальных слоев, ответственных за появление соответствующего рентгеновского максимума.
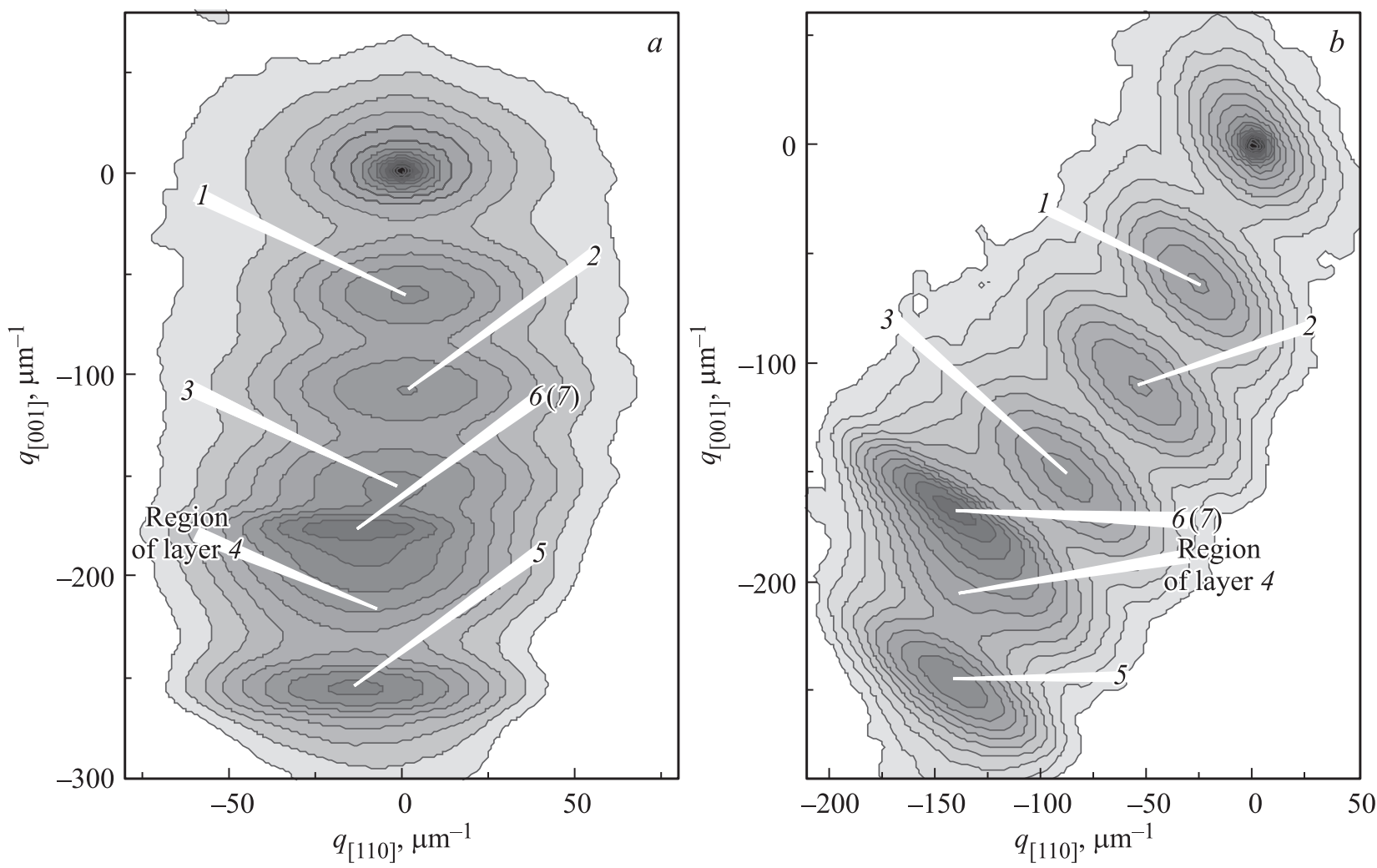

Рис. 3. Карты обратного пространства для МНЕМТ 2, полученные на основе отражений $004(a)$ и $224(b)$. Цифрами обозначены номера эпитаксиальных слоев, ответственных за появление соответствующего рентгеновского максимума. 
3.3. Деформационные поля в эпитаксиальных слоях MНЕМТ. Значения векторов обратного пространства $q_{z}^{004}, q_{y}^{004}$ и $q_{y}^{224}$ позволяют определить так называемые „полные“ деформации (относительно подложки GaAs) эпитаксиальных слоев исследованных МНЕМТ вдоль кристаллографических осей [001] и [110]: $\left[\left(a_{\perp}-a_{s}\right) / a_{s}\right]_{[001]}$ и $\left[\left(a_{\|}-a_{s}\right) / a_{s}\right]_{[110]}$, где и $a_{\perp}$ и $a_{\|}-$ соответственно вертикальный и латеральный периоды решетки слоя, $a_{s}$ - период решетки подложки. В соответствии с [19]

$$
\begin{gathered}
\left(\frac{a_{\perp}-a_{s}}{a_{s}}\right)_{[001]}=-\frac{q_{z}^{004}}{\left(4 / a_{s}\right)+q_{z}^{004}}, \\
\left(\frac{a_{\|}-a_{s}}{a_{s}}\right)_{[110]}=-\frac{Q_{y}^{224}}{\left(2^{3 / 2} / a_{s}\right)+Q_{y}^{224}},
\end{gathered}
$$

где

$$
Q_{y}^{224}=q_{y}^{224}-q_{y}^{004} .
$$

Выражение (3) учитывает эффект пространственной разориентации эпитаксиальных слоев относительно подложки GaAs. В случае отсутствия недиагонального компонента тензора упругой деформации $e_{12}$ и равенства упругих деформаций $e_{11}$ и $e_{22}$ (эти условия в настоящей работе принимаются a priori) величины $\left[\left(a_{\perp}-a_{s}\right) / a_{s}\right]_{[001]}$ и $\left[\left(a_{\|}-a_{s}\right) / a_{s}\right]_{[110]}$ соответствуют полным деформациям $\varepsilon_{33}$ и $\varepsilon_{11}$ вдоль главных кристаллографических осей (заметим, что при вращении кубической решетки на угол $45^{\circ}$ вокруг оси [001] операции, необходимой для перехода от оси [110] к оси [100], - сформулированные выше условия обеспечивают равенство между величинами $\left[\left(a_{\|}-a_{s}\right) / a_{s}\right]_{[110]}$ и $\left.\varepsilon_{11}\right)$. Величины $\varepsilon_{33}$ и $\varepsilon_{11}$ позволяют на основе линейной теории упругости рассчитать параметр рассогласования $\varepsilon_{0}=\left(a_{R}-a_{s}\right) / a_{s}$, где $a_{R}-$ период полностью релаксированной решетки данного эпитаксиального слоя.

Простейшим способом расчета остаточных упругих деформаций в эпитаксиальных слоях многослойных тонкопленочных систем является представление о том, что в силу генерации на межфазных границах эпитаксиальных слоев дислокаций несоответствия и протекания вследствие этого пластической деформации, упругие напряжения $\sigma_{33}$ в каждом эпитаксиальном слое исследованных МНЕМТ отсутствуют. В этом случае для кристаллов кубической сингонии между упругими деформациями $e_{11}$ и $e_{33}$ в соответствии с законом Гука будет справедливым следующее соотношение:

$$
\frac{e_{33}}{e_{11}}=-\frac{2 C_{12}}{C_{11}}
$$

где $C_{11}$ и $C_{12}$ - коэффициенты упругой жесткости. Упругие деформации $e_{11}$ и параметр рассогласования $\varepsilon_{0}$ в кристаллах кубической сингонии связаны между собой соотношением [20]

$$
e_{i i}=\varepsilon_{i i}-\varepsilon_{0},
$$

которое позволяет определить упругие деформации относительно подложки в каждом эпитаксиальном слое.
Учитывая выражения (4) и (5), можно показать, что параметр рассогласования $\varepsilon_{0}$ и полные деформации $\varepsilon_{33}$ и $\varepsilon_{11}$ (величины, определяемые экспериментально) связаны между собой следующим образом:

$$
\varepsilon_{0}=\varepsilon_{33}-\frac{2 C_{12}}{C_{11}+2 C_{12}}\left(\varepsilon_{33}-\varepsilon_{11}\right) .
$$

Результаты расчета $\varepsilon_{0}^{(n)}$ и $e_{11}^{(n)}(n-$ номер слоя $)$, выполненные на основе экспериментальных данных, для обеих MHЕMT представлены в табл. 3. Период решетки GaAs был принят в соответствии с [21] равным $0.565321 \mathrm{~nm}$. Необходимые при расчетах значения коэффициентов упругой жесткости тройных растворов $\mathrm{In}_{x} \mathrm{Al}_{1-x}$ As $C_{11}$ и $C_{12}$ были получены на основании закона Вегарда исходя из соответствующих данных для химических соединений InAs и AlAs [22]. Приведенные в табл. 3 значения $\varepsilon_{0}^{(4)}$ и $e_{11}^{(4)}$ для МНЕМТ 2 были определены на основе экспериментально установленной для этой гетероструктуры линейной зависимости $\varepsilon_{0}$ от расстояния данной ступени до подложки, а также с учетом выражений (5) и (6). Пространственные распределения параметра рассогласования $\varepsilon_{0}$ для обеих МНЕМТ приведены на рис. 4.
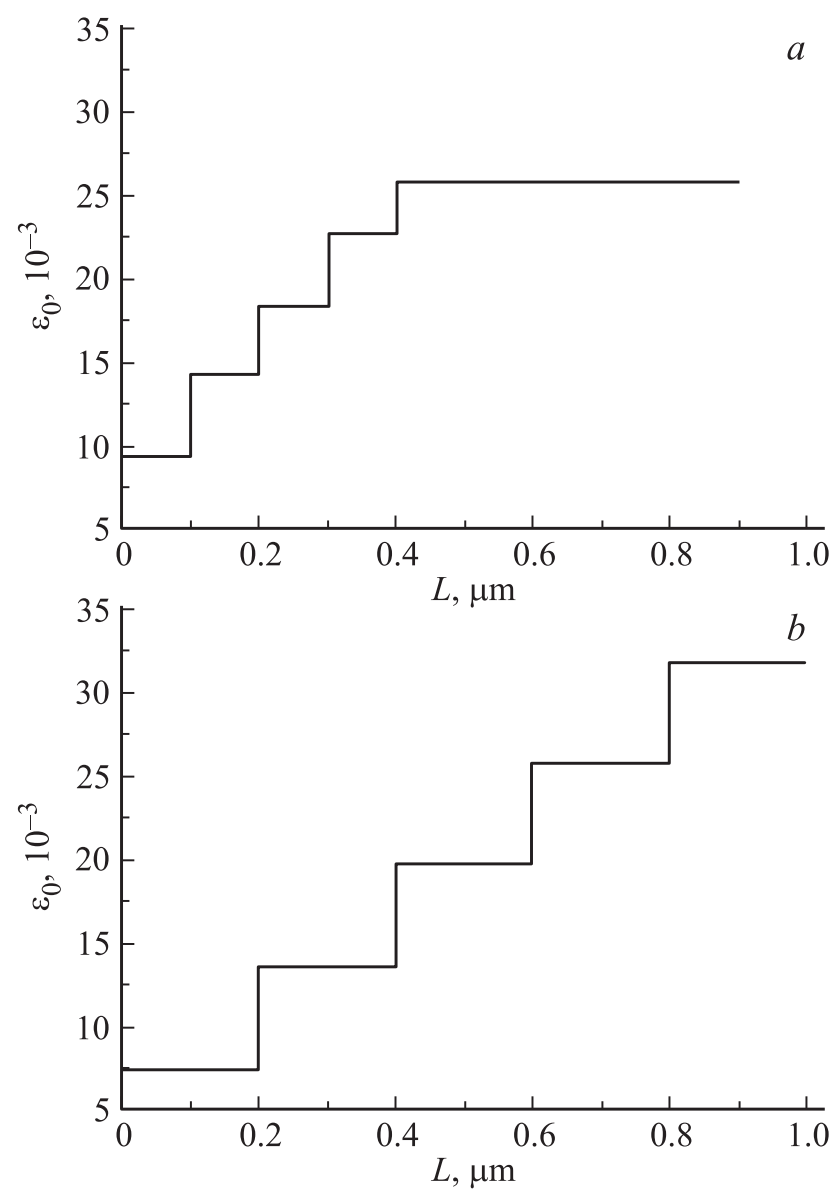

Рис. 4. Пространственные распределения параметра рассогласования $\varepsilon_{0}$ в эпитаксиальных слоях, испытывающих деформационное сжатие в МНEMT $1(a)$ и MHEMT $2(b)$. $L-$ расстояние до подложки. 
Таблица 3. Параметр рассогласования и остаточная упругая деформация в слоях МНЕМТ 1 и 2

\begin{tabular}{c|c|r|c|c}
\hline $\begin{array}{c}\text { Гетеро- } \\
\text { структура }\end{array}$ & $\begin{array}{c}\text { Номер } \\
\text { слоя }\end{array}$ & $\varepsilon_{0}, 10^{-3}$ & $e_{11}, 10^{-3}$ & $\begin{array}{c}\text { Толщина элемента } \\
\text { конструкции, } \mu \mathrm{m}\end{array}$ \\
\hline MHЕMT 1 & 1 & 9.444 & -1.38 & 0.1 \\
& 2 & 14.342 & -1.71 & 0.1 \\
& 3 & 18.385 & -1.74 & 0.1 \\
& 4 & 22.686 & -1.26 & 0.1 \\
MHEMT 2 & $5(6,7)$ & 25.801 & -4.01 & 0.5 \\
& 1 & 7.359 & -1.45 & 0.2 \\
& 2 & 13.299 & -2.19 & 0.2 \\
& 4 & 19.833 & -1.96 & 0.2 \\
& 5 & 25.778 & -1.39 & 0.2 \\
& $6(7)$ & 25.854 & -5.68 & 0.2 \\
& & & 0.28 & 0.4
\end{tabular}

Из табл. 3 видно, что в МНЕМТ 2 упругая деформация в инверсной ступени и барьерном слое по абсолютной величине на порядок меньше, чем в остальных слоях. Такое положение дел соответствует идеологии создания этого элемента конструкции ММ-буфера [23] и свидетельствует об удачном подборе концентрации In при выращивании МНЕМТ 2. В связи с этим при дальнейшем анализе полученных результатов наличием деформации в инверсной ступени и нижнем барьерном слое в МНЕМТ 2 мы пренебрегали. Актуальными слоями для анализа распределения остаточной упругой деформации в слоях МНЕМТ 2 являются пять ступеней ММ-буфера, толщина которых одинакова и равна $0.2 \mu \mathrm{m}$. МНЕМТ 1 также фактически состоит из пяти слоев, однако в этом случае слои имеют разную толщину: четыре нижних слоя имеют толщину $0.1 \mu \mathrm{m}$, а верхний (пятый) слой, объединяющий пятую ступень ММ-буфера, залечивающий и барьерный слои, имеет

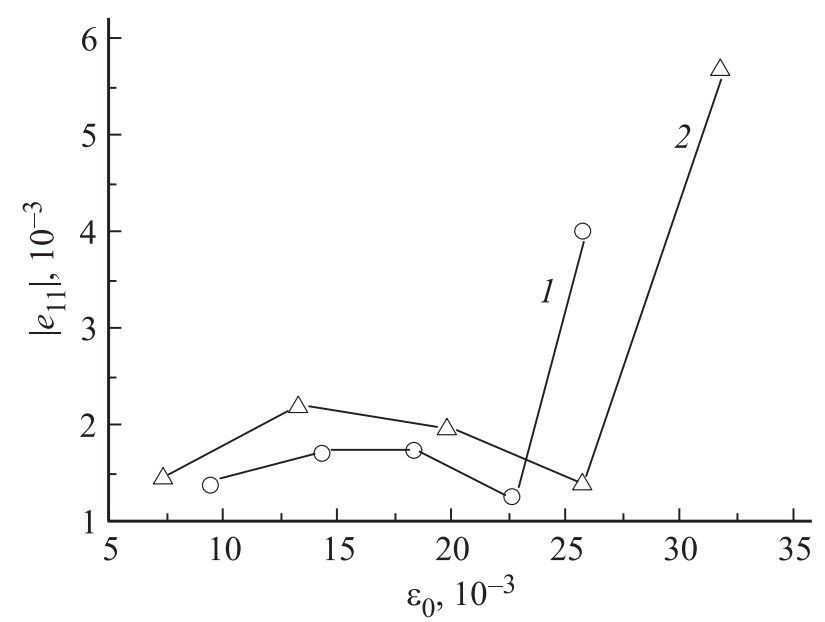

Рис. 5. Распределения остаточной упругой деформации в MHEMT, представленные в виде зависимостей $\left|e_{11}\right|$ от $\varepsilon_{0}$. Цифры 1 и 2 обозначают порядковый номер MHEMT. толщину $0.5 \mu \mathrm{m}$. Характер деформационных полей в исследованных МНЕМТ иллюстрирует рис. 5, на котором представлены зависимости $\left|e_{11}\right|$ от $\varepsilon_{0}$ (аналог пространственного распределения остаточной упругой деформации в гетероструктурах). Видно, что обе зависимости имеют одинаковый вид, причем внутренние слои ММ-буферов в обеих МНЕМТ характеризуются ненулевыми значениями упругой деформации. Это обстоятельство находится в противоречии с модельными представлениями о характере деформационных полей в ступенчатом ММ-буфере [24]. Причина возникновения упругонапряженного состояния внутренних ступеней ММ-буфера заключается в протекании в них процесса деформационного упрочнения, что, по-видимому, характерно для многослойных тонкопленочных структур, несмотря на малую толщину каждой из ступеней ММ-буферов в обеих МНЕМТ.

\section{4. Обсуждение результатов}

Полученные результаты позволяют количественно охарактеризовать деформационные поля исследованных MHЕМТ. Для верхних эпитаксиальных слоев исследованных гетероструктур выполняется следующее равенство:

$$
\begin{aligned}
& {\left[\left(e_{11}^{(5-6-7)}-e_{11}^{(4)}\right)^{2} h_{\mathrm{dfl}}\right]_{\text {MHEMT } 1}} \\
& \quad \approx\left[\left(e_{11}^{(5)}-e^{(4)_{11}}\right)^{2} h_{\mathrm{dfl}}\right]_{\text {MHEMT } 2},
\end{aligned}
$$

где $h_{\mathrm{dfl}}-$ толщина бездислокационного слоя. Для MHEMT $1 h_{\mathrm{dfl}}=0.5 \mu \mathrm{m}$, для МНЕMT $2 h_{\mathrm{dfl}}=0.2 \mu \mathrm{m}$. Произведения левой и правой частей выражения (7) равны 0.0038 и $0.0037 \mathrm{~nm}$ соответственно. Выражение (7) может быть записано в общем виде

$$
\left(e_{11}^{\mathrm{dfl}}-e_{11}^{\mathrm{ias}}\right)=\frac{k}{h_{\mathrm{dfl}}}
$$

где $e_{11}^{\mathrm{dfl}}-$ упругая деформация в бездислокационном слое, $e_{11}^{\text {ias }}-$ остаточная упругая деформация во внутренней ступени ММ-буфера, примыкающей к бездислокационному слою, $k-$ феноменологическая константа (со значением $k=0.00375 \mathrm{~nm}-$ средняя экспериментальная величина $k$ ). Обращает на себя внимание то,что по своей форме выражение (8) близко к выражению, полученному в [12] для описания структурной релаксации в однослойных гетерофазных структурах, выращенных на подложках (001) GaAs на основе тройных растворов $\operatorname{In}_{x} \mathrm{Ga}_{1-x}$ As при $x=0.1-0.4$. Согласно [12], процесс структурной релаксации в таких однослойных гетероструктурах следует закону

$$
e_{11}^{2}=\frac{k}{h}
$$

где $h$ - толщина эпитаксиального слоя. Феноменологическая константа $k$ в выражении (9) равна $0.0037 \mathrm{~nm}$. 
Таким образом, выражения (8) и (9) оперируют одним и тем же значением $k$, что свидетельствует об универсальности феноменологического подхода, основанного на существовании энергетического предела состояния упругодеформированной среды, для описания процесса структурной релаксации. Уравнение (8) можно рассматривать как распространение выражения (9) на случай двухслойной системы, в которой роль подложки выполняет бездислокационный слой. Принципиальным отличием этого случая от случая однослойной гетероструктуры является тот факт, что процесс структурной релаксации происходит в соседнем нижележащем слое, в то время как бездислокационный слой испытывает сжатие. Поскольку бездислокационный слой существенно тоньше массивной подложки в однослойных гетероструктурах, он более восприимчив к процессам структурной релаксации, протекающим в нижележащей ступени ММ-буфера. Оба слоя - бездислокационный слой и примыкающий к нему эпитаксиальный слой (нижерасположенная ступень ММ-буфера) - представляют собой единую уравновешенную систему, в которой величина упругой деформации в бездислокационном слое выше по сравнению с остаточной упругой деформацией в однослойной гетероструктуре с эпитаксиальным слоем равной толщины. Данное обстоятельство отражает взаимосвязь между этими двумя элементами ММ-буфера и является, по-видимому, необходимым условием формирования бездислокационного слоя. Выявление взаимосвязи значений упругих деформаций для всех внутренних ступеней ММ-буфера требует дальнейшего изучения.

\section{5. Заключение}

Выполненное исследование свидетельствует о возможности распространения феноменологического подхода, описывающего процесс структурной релаксации в однослойных эпитаксиальных гетероструктурах, на многослойные тонкопленочные системы, что позволяет определить предельную величину упругой деформации в бездислокационном слое метаморфного ступенчатого буфера. В численном отношении данный феноменологический закон выражается обратной квадратнокорневой зависимостью величины упругой деформации бездислокационного слоя от его толщины. Отличие описания деформационного поля в многослойной системе от описания в случае однослойной гетероструктуры заключается в необходимости учета остаточной упругой деформации в нижележащем слое, на котором выращивается бездислокационный слой. Остаточная деформация нижележащего слоя оказывает существенное влияние на величину упругой деформации бездислокационного слоя, увеличивая ее значение по сравнению с остаточной упругой деформацией в эпитаксиальном слое равной толщины в однослойной гетероструктуре.

\section{Список литературы}

[1] M. Imaizumi, M. Hirotani, T. Soga. Proc. of IEEE $42^{\text {nd }}$ Photovoltaic Specialist Conf. New Orleans, USA (2015). 5 p.

[2] R. Kumar, A. Bag, P. Mukhopadhyay, S. Das, D. Biswas. Appl. Surf. Sci. 357, 922 (2015).

[3] T. Kujofsa, J.E. Ayers. Int. J. High Speed Electron. Systems 24, 152009 (2015).

[4] A.M. Andrews, J.S. Speck, A.E. Romanov, M. Bobeth, W. Pompe. J. Appl. Phys. 91, 1933 (2002).

[5] Г.Б. Галиев, Е.А. Климов, Р.М. Имамов, Г.В. Ганин, С.С. Пушкарев, П.П. Мальцев, О.М. Жигалина, А.С. Opeхов, А.Л. Васильев, М.Ю. Пресняков, И.Н. Трунькин. Поверхность. Рентгеновские, синхротронные и нейтронные исследования. 5, 32 (2016).

[6] J. Tersoff. Appl. Phys. Lett. 62, 693 (1993).

[7] D.J. Dunstan. J. Mater. Sci.: Mater. Electron. 8, 337 (1997).

[8] E. Ayers. Heteroepitaxy of semiconductors. Theory, growth, and characterization. Taylor and Francis Group, N.Y. (2007). $439 \mathrm{p}$.

[9] D.J. Dunstan, P. Kidd, L.K. Howard, R.H. Dixon. Appl. Phys. Lett. 59, 3390 (1991).

[10] D.J. Dunstan, P. Kidd, P.F. Fewster, N.L. Andrew, R. Grey, P.R. David, L. Gonzalez, Y. Gonzalez, A. Sacedon, F. Gonzalez-Sanz. Appl. Phys. Lett. 65, 839 (1994).

[11] D.J. Dunstan, S. Young, R.H. Dixon. J. Appl. Phys. 70, 3039 (1991).

[12] A.V. Drigo, A. Audinli, A. Carnera, F. Genova, C. Rigo, C. Ferrari, P. Franzosi, G. Salviati. J. Appl. Phys. 66, 1975 (1989).

[13] K. Kamigaki, H. Sakashita. M. Nakayama, N. Sano, H. Terauchi. Appl. Phys. Lett. 49, 1071 (1986).

[14] P.J. Orders, B.F. Usher. Appl. Phys. Lett. 50, 980 (1987).

[15] P.M.J. Maree, J.C. Barbour, J.F. van der Veen, K.L. Kavanagh, C.W.T. Bulle-Lieuwma, M.P.A. Viegers. J. Appl. Phys. 62, 4413 (1987).

[16] Д.К. Боуэн, Б.К. Таннер. Высокоразрешающая рентгеновская дифрактометрия и топография. Наука, СПб. (2002). $275 \mathrm{c}$.

[17] В.А. Бушуев, Р.Н. Кютт, Ю.П. Хапачев. Физические принципы рентгенодифрактометрического определения параметров реальной структуры многослойных эпитаксиальных пленок. Кабардино-Балкарский гос. ун-т, Нальчик (1996). $181 \mathrm{c}$.

[18] А.Н. Алёшин, А.С. Бугаев, М.А. Ермакова, О.А. Рубан. ФТП 49, 1065 (2015).

[19] J.-M. Chauveau, Y. Androussi, A. Lefebvre, J. Di Persio, Y. Cordier. J. Appl. Phys. 93, 4219 (2003).

[24] Ю.П. Хапачев, Ф.Н. Чуховский. Кристаллография 34, 776 (1989).

[21] С.С. Стрельченко, В.В. Лебедев. Соединения $A_{3} B_{5}$. Справочник. Металлургия, М. (1984). 144 с.

[22] K.N. Tu, J.W. Mayer, L.C. Feldman. Electronic thin film science. For electrical engineers and materials scientists. Macmillan Publishing Company, N.Y. (1992). 428 p.

[23] Y. Cordier, D. Ferre. J. Cryst. Growth 201/202, 263 (1999).

[24] D.J. Dunstan. Phil. Mag. A 73, 1323 (1996). 